\title{
Pleural Fluid: Visual Appearance and Etiology
}

\author{
Taylor Warmoth BS, Gilbert Berdine MD
}

Every thoracentesis report should include a visual impression of the gross appearance of the fluid. The use of the term 'sero-sanguineous' is used so universally as to render it meaningless. The color and clarity of pleural fluid can suggest what caused the pleural effusion, but there seem to be few terms to describe the appearance of pleural fluid color. An observant practitioner of thoracentesis cannot help but appreciate the similarity between pleural fluid and another of nature's fluids: beer.

The color of beer, like pleural fluid, depends on

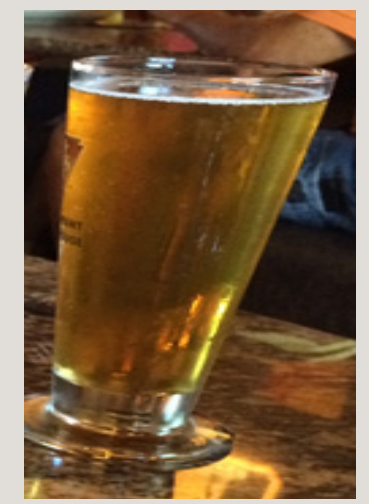

Beer one: BJ's Brewhouse Blonde ${ }^{\circ}$, a Kölsch style pale beer, is comparable in color to a Coors Light. This color in pleural fluid is a classic representation of a transudative effusion resulting from congestive heart failure. The transudation of fluid is due to increased pressure driving a protein-free fluid across an intact barrier between capillary and pleural space. Note the clarity and lack of foam in this beer.

Corresponding author: Taylor Warmoth

Contact Information: taylor.warmoth@ttuhsc.edu DOI: 10.12746/swrccc 2016.0413.171 a number of factors. For beer, these factors include boil times and the coupling of amino acids to make sugars. And like beer, the color of pleural fluid is a direct result of the process that made it. Comparing pleural fluid to types of beer provides the opportunity to better understand its characteristics and the underlying cause of its production. For this classification system, four beers will be used as descriptors: 1. BJ's Brewhouse Blonde $®$, a Kölsch style pale beer; 2. BJ's Harvest Hefeweizen $₫$, a wheat beer; 3. BJ's Oasis ${ }^{\circledR}$ Amber, an American amber or red ale; and 4. BJ's Tatonka® Stout, a Russian imperial stout.

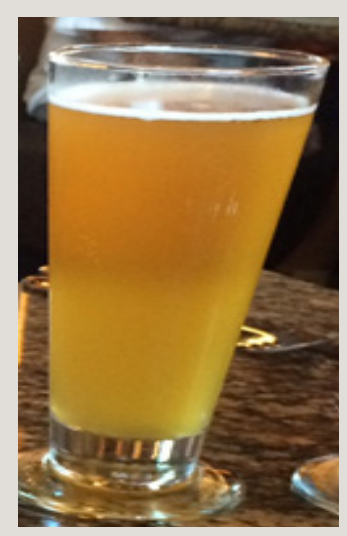

Beer two: BJ's Harvest Hefeweizen $\circledR$, a wheat beer, is much cloudier but is still light in color. A pleural effusion of this color could represent a CHF patient in whom the pleural effusion was allowed to sit for some time before thoracentesis. Much more likely, the greater amount of foam in the head of the beer and the increased turbidity (reduced clarity) indicate a higher protein content, much like an exudative effusion resulting from an inflammatory process. Inflammatory processes damage the barrier leading to leakage of protein and cellular material into the pleural space. 


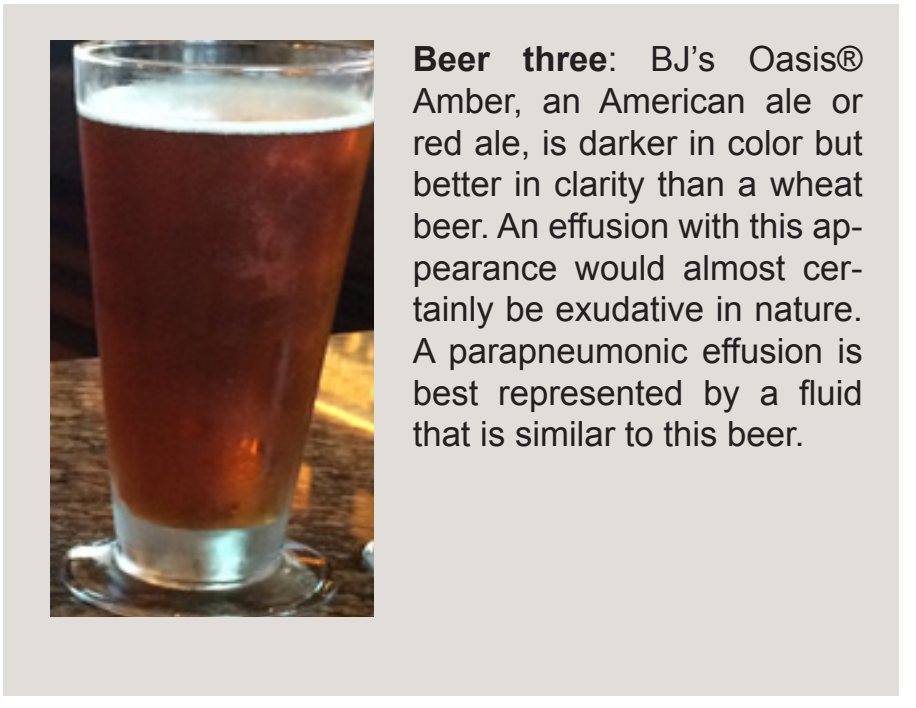

While not a conventional classification system, these comparisons of pleural fluid to beers provide a more useful description of gross appearance than the ubiquitous term 'sero-sanguineous' which covers the entire spectrum from serous to sanguineous and provides no useful hints about cause.

Author affiliations : Taylor Warmoth is a medical student at Texas Tech University Health Sciences Center, Lubbock, TX. Gilbert Berdine is a faculty member in the Department of Internal Medicine at TTUHSC, Lubbock, TX.

Submitted: $11 / 1 / 2015$

Accepted: $12 / 8 / 2015$

Reviewers: Sam Copeland MD, Michael Phy DO

Conflict of Interest: None

Published electronically: 1/15/2016

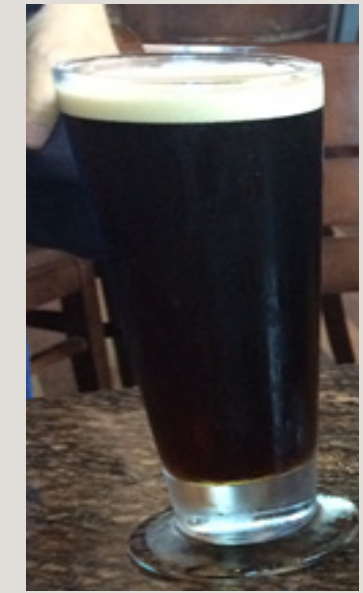

Beer four: BJ's Tatonka $\circledR$ Stout, a Russian imperial stout that is dark in color and high in protein. This would be similar to a grossly bloody effusion resulting from a malignancy. It could also represent the remnants of a pulmonary embolism with infarction. The dark color is due to lysis of red blood cells over time. Red blood cell lysis also causes a very high LDH level in the pleural fluid. 